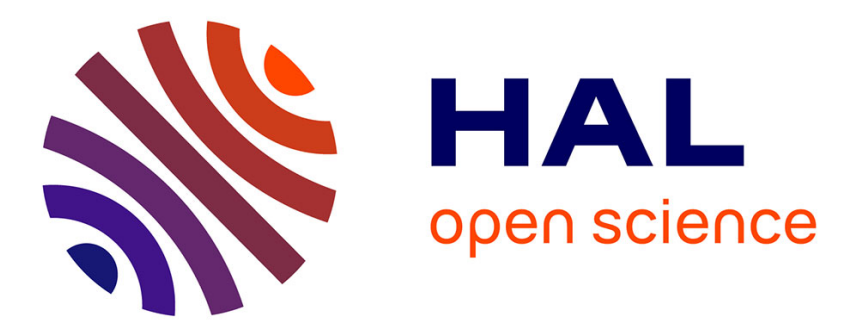

\title{
Vibration analysis of cable-driven parallel robots based on the dynamic stiffness matrix method
}

\author{
Huan Yuan, Eric Courteille, Marc Gouttefarde, Pierre-Elie Hervé
}

\section{To cite this version:}

Huan Yuan, Eric Courteille, Marc Gouttefarde, Pierre-Elie Hervé. Vibration analysis of cable-driven parallel robots based on the dynamic stiffness matrix method. Journal of Sound and Vibration, 2017, 394, pp.527-544. 10.1016/j.jsv.2017.02.003 . hal-01484736

\section{HAL Id: hal-01484736 \\ https://hal-univ-rennes1.archives-ouvertes.fr/hal-01484736}

Submitted on 4 Jul 2017

HAL is a multi-disciplinary open access archive for the deposit and dissemination of scientific research documents, whether they are published or not. The documents may come from teaching and research institutions in France or abroad, or from public or private research centers.
L'archive ouverte pluridisciplinaire HAL, est destinée au dépôt et à la diffusion de documents scientifiques de niveau recherche, publiés ou non, émanant des établissements d'enseignement et de recherche français ou étrangers, des laboratoires publics ou privés. 


\title{
Vibration analysis of cable-driven parallel robots based on the dynamic stiffness matrix method
}

\author{
Han Yuan ${ }^{\mathrm{a}, \mathrm{b}}$, Eric Courteille ${ }^{\mathrm{b}, *}$, Marc Gouttefarde $^{c}$, Pierre-Elie Hervé ${ }^{c}$ \\ a School of Mechanical Engineering and Automation, Harbin Institute of Technology Shenzhen, Xili, Nanshan District, \\ Shenzhen Zip Code 518055, PR China \\ ${ }^{\mathrm{b}}$ Université Européenne de Bretagne, INSA-LGCGM-EA 3913, 20, Avenue des Buttes de Cöesmes, 35043 Rennes Cedex, France \\ ${ }^{\mathrm{c}}$ LIRMM - CNRS - Université de Montpellier - Robotics Department, 161 rue Ada, 34095 Montpellier Cedex 5, France
}

This paper focuses on the vibration analysis of Cable-Driven Parallel Robots (CDPRs). An oscillating model of CDPRs able to capture the dynamic behavior of the cables is derived using Lagrangian approach in conjunction with the Dynamic Stiffness Matrix method. Then, an original approach to analyze the modal interaction between the local cable modes and the global CDPR modes is presented. To illustrate this approach, numerical investigations and experimental analyses are carried out on a large-dimension 6-DOF suspended CDPR driven by 8 cables.

\section{Introduction}

A parallel robot can be defined as a closed-loop kinematic chain mechanism whose end-effector is linked to the base by several independent kinematic chains [1].

Cable-driven parallel robots (CDPRs) are a special variant of traditional rigid-link parallel robots such as the Stewart platform [2] and the Delta robot [3]. Flexible cables are used instead of rigid links to connect the movable end-effector and the fixed base. The end-effector is manipulated by changing the lengths of the cables by means of winches.

CDPRs have several advantages. First, CDPRs can achieve large workspaces. The cables being flexible, large cable lengths can easily be released and retracted. Thus, cables allow much larger ranges of motion compared to conventional rigid-links. CDPRs can be designed to be very large with an acceptable cost, such as the Skycam ${ }^{1}$, and the Five hundred meter Aperture Spherical Telescope (FAST) [4].

In addition, CDPRs have high energy efficiency and large payload-to-weight ratios since they use lightweight cables and usually have stationary heavy components and few moving parts.

Another advantage of CDPRs is their simple structure. They can be relatively easily disassembled, transported, reassembled, and reconfigured which makes them suitable for search and rescue applications [5-7]. Last but not least, since

\footnotetext{
* Corresponding author.

E-mail addresses: han.yuan@insa-rennes.fr (H. Yuan), eric.courteille@insa-rennes.fr (E. Courteille), Marc.Gouttefarde@lirmm.fr (M. Gouttefarde), Pierre-Elie.Herve@lirmm.fr (P.-E. Hervé).

${ }^{1}$ Skycam is a product of Skycam company: http://www.skycam.tv/.
} 




cables are flexible, interferences between cables and/or collisions between cables and other objects in the operating environment may cause less accident or damage [8,9], which is notably useful for haptic devices, such as the NEREBOT [10] and the STING-MAN [11].

Due to the compliance of cables, vibrations can become a crucial concern for CDPRs. Vibrations have a significant effect on the static and dynamic behaviors of CDPRs, such as on the positioning accuracy, settling time, trajectory tracking, as well as on force distribution and control $[1,12,13]$. Although there are a lot of previous works on the vibration analysis and control of rigid-link parallel robots, e.g. [14-22], only few studies are dedicated to the vibration analysis of CDPRs [23-30]. Vibrations can notably be induced by (brutal) end-effector velocity changes, wind disturbance, and/or friction of the cables around pulleys [24]. In applications requiring high performances, especially dynamic performances, e.g. [13,31], or in the presence of wind, e.g. [4,32], vibrations are an issue since they can affect the positioning accuracy of the end-effector, and yield fluctuations around a desired nominal end-effector trajectory.

Cables have been modeled as linear massless axial springs, and end-effector vibrations caused by axial and transverse cable flexibilities have been analyzed in simulations in [23,26]. Using the same cable model, vibration characteristics of a CDPR for processing applications are presented in [27] while, in [28], a new approach to compensate for the rotational oscillations of the end-effector using reaction wheels is proposed. [23,26-28] only consider cable elasticity, while neglecting the effect of cable mass on the cable dynamics. Although the effect of cable weight on the static cable profile has been considered in a number of works, e.g. [33-36], the effect of cable mass on the cable dynamics is totally ignored in the computation of the CDPR eigenfrequencies. An important issue of the dynamic analysis of CDPRs is to find out whether the cable natural modes and induced vibrations affect the dynamics of CDPRs. Finite Element Method (FEM) has been used in the modeling of cable dynamics $[24,25,37,38]$. Using FEM, the end-effector vibrations and the system eigenfrequencies have been studied in simulations in [24,25]. However, cable modeling based on FEM uses distributed point mass and ideal lines between them to simulate a continuous cable. Hence, it leads to a complex system with many partial differential equations. Moreover, as well-known, the accuracy of FEM depends on the number of elements so that there exists a strong trade-off between accuracy and computational complexity. The dynamic behavior of CDPRs with long sagging cables has been recently investigated in an analytical way using Hamilton's principle [30]. The assumed mode method is used to solve the obtained time-varying partial differential equations of motion. In the assumed mode method, the shape functions are a linear combination of the eigenfunctions of the related simpler problem of transverse cable vibrations with standard boundary conditions. The accuracy of the dynamic model thereby obtained highly depends on the adopted shape functions of the transverse cable vibrations and on their number. In [30], no experimental validation of the theoretical predictions of the CDPR dynamic responses has been presented. Moreover, it should also be noted that the time integration of such dynamic models has not received particular attention. There are two primary issues in time integrating these dynamic models. First, the discrete time-step integration method used for solving the dynamic equations should be carefully selected 


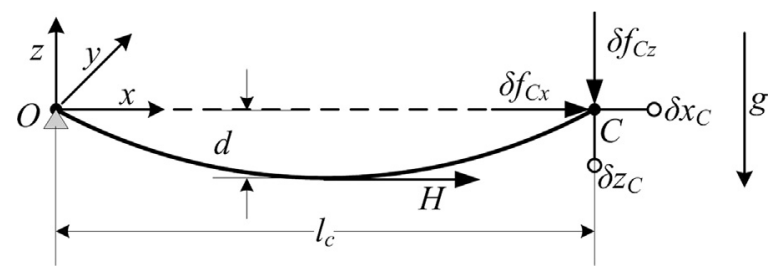

Fig. 1. Diagram of a horizontal sagging cable [39].

to ensure convergence. Second, the constitutive law governing the damping mechanisms should be carefully defined. The latter is an important issue to predict vibration levels along a trajectory, in transient responses, or in other characteristics that are influenced by energy dissipation.

Besides, an oscillating model developed using Lagrangian approach in conjunction with the Dynamic Stiffness Matrix (DSM) method was presented in our previous work [29]. This modeling framework does not allow the computation of the time-domain response (motion) of the CDPR along a trajectory as the aforementioned modeling methods, but its advantages include mathematical convenience, simplicity in numerical implementation and computation. This model is able to capture the effect of cable dynamics on the robot vibrations in the framework of the linear theory of cable vibrations [39]. The DSM method is often regarded as an exact method which provides better accuracy compared to the FEM [40-42] since it relies on the frequency-dependent shape functions that are exact solutions of the governing differential equations. Since the shape functions used in the DSM method are inherently frequency-dependent, the CDPR dynamics is analyzed in the frequency domain. This is an essential prerequisite before studying CDPR responses in the time domain.

In this paper, an original methodology is presented to analyze the modal interaction between the local cable modes and the global CDPR modes. Compared to our previous work [29], with the intention to evaluate the effect of the cable natural modes on the robot vibrations, the present paper focuses on a dynamic stiffness analysis where the calculation of the Frequency Response Functions (FRFs) are explicated. The FRFs represent the input-output relationships between an excitation force and a system response (position, velocity or acceleration). Since the assessment of the damping has a minor impact on the analysis of the CDPR eigenproperties, the frequency-dependence of the system is also analyzed in terms of eigenvalues. The changes in stiffness in the region of the cable eigenfrequencies affect the eigenfrequencies of the overall robot. This results in new and numerous resonances of the system, which may be relevant for CDPRs used in wind tunnels and for outdoor CDPR applications where a periodic excitation source can be directly or indirectly applied. System modeling and the proposed vibration analysis methodology are illustrated with a 6-DOF CDPR prototype driven by 8 cables, called CoGiRo [43]. Substantial experimental investigations have been carried out around two static equilibria of the CoGiRo CDPR end-effector. The experimental analysis of an end-effector trajectory has been also performed in the frequency domain for several trajectory durations. The corresponding result comparisons illustrate the relevance of the proposed approach.

This paper is organized as follows. The DSM method and the dynamic stiffness matrix of an inclined sagging cable are recalled in Section 2. The oscillating model of CDPRs is then set up in Section 3 where the calculation of the FRFs is also detailed. In Section 4, dynamic experiments around two static equilibria and along a trajectory are performed on the CoGiRo CDPR prototype, to analyze its vibration characteristics and to validate the proposed methodology. Finally, conclusions are drawn in Section 5.

\section{Dynamic stiffness matrix of a sagging cable}

In order to consider cable dynamics in the vibration analysis of CDPRs, the dynamic stiffness matrix (DSM) of a cable with non-negligible elasticity and mass is presented in this section by using the DSM method. The DSM of a horizontal sagging cable is first presented. Then, it is extended to an inclined sagging cable.

\subsection{Dynamic stiffness matrix of a horizontal sagging cable}

Fig. 1 shows the diagram of a horizontal sagging cable, where $d$ is the sag perpendicular to the chord, $l_{c}$ is the chord length, and $H$ is the static cable tension at the section where the cable is parallel to the chord. One cable end is fixed, and an external static force is applied to the other cable end. Under the effect of both the external static force and gravity ( $g$ is the gravitational acceleration), the shape of the cable is not a straight line, but a sagging curve in the cable vertical plane.

The considered dynamic cable model is based on the linear theory of small transverse vibrations around a static equilibrium which should be the common case for CDPRs. The following assumptions are made $[39,44,45]$.

- The cable is assumed to be continuous and uniform [39], and defined by its unstrained cross section area $A$, mass per unit length $\rho$, and linear Young's modulus $E$.

- Only small displacements are admitted to meet the requirements of linear theory [46];

- Only small cable sag is allowed, i.e., the sag to span ratio $\frac{d}{l_{c}}$ is smaller than $1 / 20$ [39]; 
- Only viscous damping is taken into consideration.

The DSM of a viscous-damped small-sag horizontal cable $\mathbf{K}_{\mathrm{dyn}-\mathrm{h}}^{2 \mathrm{D}}(\omega)$ can be defined as:

$$
\left[\begin{array}{l}
\delta f_{C x} \\
\delta f_{C z}
\end{array}\right]=\mathbf{K}_{\mathrm{dyn}-\mathrm{h}}^{2 \mathrm{D}}(\omega)\left[\begin{array}{l}
\delta x_{C} \\
\delta z_{C}
\end{array}\right]
$$

where $\left[\delta f_{C x} \delta f_{C z}\right]^{T}$ and $\left[\delta x_{C} \delta z_{C}\right]^{T}$ are the vectors of the dynamic forces and displacements shown in Fig. 1. The differential notation is used to represent small changes in force and position from the static equilibrium. In linear theory, the in-plane motion of a cable is uncoupled with its out-of-plane motion [44,45]. For the sake of convenience, the DSM is firstly deduced in the cable plane, then it will be extended to 3 dimensions without major difficulty.

It is assumed that only harmonic vibrations and exponentially variable amplitude are admitted [41]. In that case, the DSM of the cable is complex valued and depends on the frequency $\omega$ of the forcing function. The DSM $\mathbf{K}_{\mathrm{dyn}-\mathrm{h}}^{2 \mathrm{D}}(\omega)$ can be formulated as [47]:

$$
\mathbf{K}_{\mathrm{dyn}-\mathrm{h}}^{2 \mathrm{D}}(\omega)=\left[\begin{array}{ll}
K_{x x}(\omega) & K_{x z}(\omega) \\
K_{z x}(\omega) & K_{z z}(\omega)
\end{array}\right],
$$

where:

$$
\begin{aligned}
& K_{x x}(\omega)=\frac{E A}{L_{e}} \frac{1}{1+\frac{\lambda^{2}}{\Omega_{C}^{2}}(\kappa-1)}, \\
& K_{x z}(\omega)=K_{z x}(\omega)=\frac{E A}{L_{e}} \frac{\frac{1}{2} \varepsilon(\kappa-1)}{1+\frac{\lambda^{2}}{\Omega_{c}^{2}}(\kappa-1)}, \\
& K_{z z}(\omega)=\frac{E A}{L_{e}} \frac{\varepsilon^{2}}{\lambda^{2}} \frac{1}{\kappa}-\frac{E A}{L_{e}} \frac{\frac{1}{4} \frac{\varepsilon^{2}}{\lambda^{2}} \Omega_{c}^{2}\left[\kappa+\frac{\lambda^{2}}{\Omega_{C}^{2}}(\kappa-1)\right]}{1+\frac{\lambda^{2}}{\Omega_{c}^{2}}(\kappa-1)} .
\end{aligned}
$$

The parameters in Eqs. (3)-(5) are:

- $\lambda^{2}=\left(\frac{\rho g l_{c}}{H}\right)^{2} \frac{E A l_{c}}{H L_{e}}$ is the fundamental cable parameter which represents the elastic stiffness relative to the sag-induced stiffness;

- $\varepsilon=\frac{\rho g l_{c}}{H}=\frac{8 d}{l_{c}}$ is the ratio between horizontal cable weight and static cable tension;

- $L_{e}=\int_{0}^{l_{c}}\left(\frac{d s}{d x}\right)^{3} d x \simeq l_{c}\left[1+8\left(\frac{d}{l_{c}}\right)^{2}\right]$ is the cable length parameter;

- $\Omega=\omega l_{c} \sqrt{\frac{\rho}{H}}$ is the dimensionless frequency parameter.

The cable is assumed to have a viscous damping behavior and the cable transverse damping per unit length $C_{c}$ is introduced through the following damping ratio:

$$
\xi=\frac{C_{c}}{2 \rho \omega} .
$$

The following auxiliary parameter is then defined:

$$
\omega_{c}=\omega \sqrt{1-2 \xi i},
$$

and the two dimensionless quantities $\Omega_{c}$ and $\kappa$ used in Eq. (3)-(5) are:

- $\Omega_{c}=\omega_{c} l_{c} \sqrt{\frac{\rho}{H}}$,

- $\kappa=\frac{\tan \left(\frac{\Omega_{c}}{2}\right)}{\left(\frac{\Omega_{c}}{2}\right)}$.

With consideration of the out-of-plane motion (motion along the $y$-axis which is perpendicular to the cable plane), the spatial DSM of a horizontal sagging cable in 3 dimensions can be expressed as: 


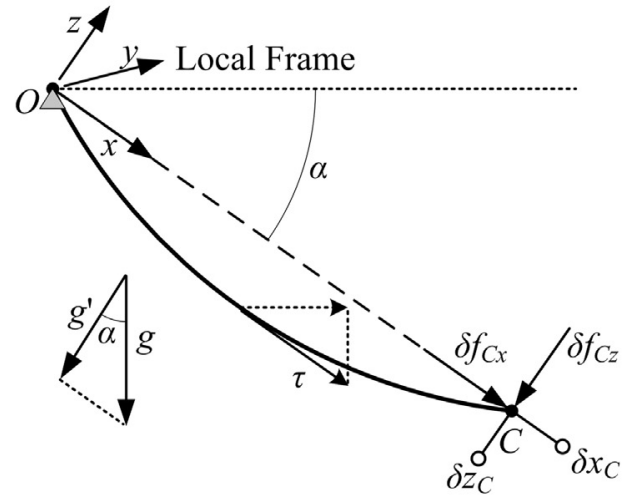

(a) In the local cable frame



(b) In the global frame

Fig. 2. Forces and displacements of an inclined sagging cable.

$$
\mathbf{K}_{\mathrm{dyn}-\mathrm{h}}^{3 \mathrm{D}}(\omega)=\left[\begin{array}{ccc}
K_{x x}(\omega) & 0 & K_{x z}(\omega) \\
0 & K_{y y}(\omega) & 0 \\
K_{z x}(\omega) & 0 & K_{z z}(\omega)
\end{array}\right] .
$$

Due to the uncoupling between the in-plane and the out-of-plane motions, the interaction coefficients in Eq. (8) are zeros. According to [44], the stiffness matrix coefficient for the out-of-plane motion is $K_{y y}(\omega)=\frac{\tau\left(4-\kappa^{2} \Omega_{c}^{2}\right)}{4 l_{c}}$. The other coefficients are the same as those in Eq. (2).

\subsection{Dynamic stiffness matrix of an inclined sagging cable}

The dynamic analysis of a horizontal sagging cable presented in Section 2.1 can be extended to study the dynamics of an inclined sagging cable $[39,48]$.

Fig. 2a represents an inclined sagging cable in the local cable frame, where the $x$-axis is parallel to the chord. It can be obtained by rotating the horizontal sagging cable (Fig. 1) about the $y$-axis, where the rotation angle is $\alpha$. The extension to an inclined sagging cable is made by the following substitutions. Firstly, the gravity acceleration $g$ is replaced by the gravitational component $g^{\prime}$, where $g^{\prime}$ is perpendicular to the cable chord and $g^{\prime}=g \cdot \cos \alpha$. Secondly, the horizontal static cable tension $H$ is replaced by $\tau$. $\tau$ represents the static cable tension at the section where the cable is parallel to the chord. Parameters related to $H$ become:

$$
\begin{aligned}
& \lambda^{2}=\left(\frac{\rho g l_{c}}{\tau}\right)^{2} \frac{E A l_{c}}{\tau L_{e}} \cos ^{2} \alpha, \\
& \varepsilon=\frac{\rho g l_{c}}{\tau} \cos \alpha=\frac{8 d}{l_{c}}, \\
& \Omega=\omega l_{c} \sqrt{\frac{\rho}{\tau}}, \\
& \Omega_{c}=\omega_{c} l_{c} \sqrt{\frac{\rho}{\tau}} .
\end{aligned}
$$

With these new definitions, the theory for the vibration analysis of a horizontal sagging cable can be used for an inclined sagging cable. It should be noted that according to [39,48], this extension is valid as long as the cable parameters $\lambda^{2}$ and $\varepsilon$ together with the inclination angle $\alpha$ do not exceed certain limits. In particular, $\lambda^{2}$ should maintain a proper distance (about $20 \%)$ from the so-called crossover points $4 n^{2} \pi^{2}(n=1,2 \ldots)$, specifically:

$$
\begin{aligned}
& \lambda^{2} \leq 24, \\
& \text { and } \alpha \leq 60^{\circ}, \quad \varepsilon \leq 0.1\left(\frac{d}{l_{c}} \leq \frac{1}{80}\right)
\end{aligned}
$$




$$
\text { or } \alpha \leq 30^{\circ}, \varepsilon \leq 0.24\left(\frac{d}{l_{c}} \leq \frac{1}{33}\right) \text {. }
$$

The DSM can now be expressed in the global cable frame. Fig. $2 \mathrm{~b}$ represents the forces and displacements of an inclined sagging cable in the global cable frame $\mathfrak{R}_{i}$, where the $z_{i}$-axis is vertical and directed upward; $O x_{i} z_{i}$ is in the cable plane. This global cable frame can be obtained by rotating the local cable frame (in Fig. 2a) $\alpha$ degrees around the $y$-axis. The global DSM ${ }^{\mathrm{i}} \mathbf{K}_{\text {dyn }}^{2 \mathrm{D}}(\omega)$ is defined as the DSM in the global cable frame $\mathfrak{R}_{i}$, i.e.:

$$
\left[\begin{array}{l}
{ }^{i} \delta f_{C X} \\
{ }^{i} \delta f_{C z}
\end{array}\right]={ }^{i} \mathbf{K}_{\mathrm{dyn}}^{2 \mathrm{D}}(\omega)\left[\begin{array}{l}
{ }^{i} \delta x_{C} \\
{ }^{i} \delta z_{C}
\end{array}\right]
$$

where $\left[{ }^{i} \delta f_{C X}{ }^{i} \delta f_{C z}\right]^{T}$ and $\left[{ }^{i} \delta x_{C}{ }^{i} \delta z_{C}\right]^{T}$ are the vectors of the dynamic forces and displacements around a static equilibrium as shown in Fig. 2b. The relationships between $\left[\delta f_{C X} \delta f_{C z}\right]^{T}$ and $\left[{ }^{i} \delta f_{C x}{ }^{i} \delta f_{C z}\right]^{T}$, and between $\left[\delta x_{C} \delta z_{C}\right]^{T}$ and $\left[{ }^{i} \delta x_{C}{ }^{i} \delta z_{C}\right]^{T}$ can be expressed by means of the rotation matrix ${ }^{\mathbf{i}} \mathbf{T}$ that maps coordinates in the local cable frame to coordinates in the global cable frame:

$$
\begin{gathered}
{\left[{ }^{i} \delta f_{C X}{ }^{i} \delta f_{C z}\right]^{T}={ }^{\mathbf{i}} \mathbf{T}\left[\delta \mathbf{f}_{C X} \delta \mathbf{f}_{C z}\right]^{T},} \\
{\left[{ }^{i} \delta x_{C}{ }^{i} \delta z_{C}\right]^{T}={ }^{\mathbf{i}} \mathbf{T}\left[\delta \mathbf{x}_{C} \delta \mathbf{z}_{C}\right]^{T},}
\end{gathered}
$$

where ${ }^{\mathbf{i}} \mathbf{T}=\left[\begin{array}{c}\cos \alpha \sin \alpha \\ -\sin \alpha \cos \alpha\end{array}\right]$. Therefore, the DSM of an inclined sagging cable in the global cable frame $\mathfrak{R}_{i}$ can be obtained as:

$$
{ }^{\mathrm{i}} \mathbf{K}_{\mathrm{dyn}}^{2 \mathrm{D}}(\omega)={ }^{\mathbf{i}} \mathbf{T} \mathbf{K}_{\mathrm{dyn}}^{2 \mathrm{D}}(\omega)^{\mathbf{i}} \mathbf{T}^{-1} \text {. }
$$

where $\mathbf{K}_{\mathrm{dyn}}^{2 \mathrm{D}}(\omega)$ is defined as in Eq. (2) but with $\lambda^{2}, \varepsilon, \Omega$, and $\Omega_{c}$ defined in Eq. (9)-(12).

In Eq. (18), $\mathbf{K}_{\mathrm{dyn}}^{2 \mathrm{D}}(\omega)$ is a $2 \times 2$ matrix that only considers the in-plane cable motion. If the out-of-plane cable motion is taken into account, the $3 \times 3$ DSM of an inclined sagging cable in the global frame can be obtained through a similar coordinate transformation:

$$
{ }^{\mathrm{i}} \mathbf{K}_{\mathrm{dyn}}^{3 \mathrm{D}}(\omega)=\left[\begin{array}{ccc}
\cos \alpha & 0 & \sin \alpha \\
0 & 1 & 0 \\
-\sin \alpha & 0 & \cos \alpha
\end{array}\right] \cdot \mathbf{K}_{\mathrm{dyn}}^{3 \mathrm{D}}(\omega) \cdot\left[\begin{array}{ccc}
\cos \alpha & 0 & \sin \alpha \\
0 & 1 & 0 \\
-\sin \alpha & 0 & \cos \alpha
\end{array}\right]^{-1} .
$$

\section{Oscillating model and vibration analysis of CDPRs}

Based on the DSM of an inclined sagging cable presented in Section 2, the oscillating model of CDPRs is presented in this section, with consideration of the rigid-body vibrations of the end-effector suspended on the cable stiffness, the local cable vibrations, and the coupling between both. Firstly, the DSM of CDPRs is formulated. Then, the oscillating model of the endeffector around a static equilibrium is formulated through the Lagrange's equations. Finally, FRFs under a harmonic excitation are defined. By means of the plots of the FRFs, the resonances of CDPRs can be identified and the effect of cable dynamics on the overall system dynamic behavior can be analyzed.

\subsection{Computation of the dynamic stiffness matrix of CDPRs}

For CDPRs, the system stiffness is mainly affected by the stiffness of their cables, actuators and end-effector. By assuming that the compliance of the end-effector and the actuators is negligible compared to the compliance of the driving cables, in the oscillating model, the end-effector is considered as a rigid-body suspended on the stiffness of the cables. The oscillations of the end-effector around a static equilibrium are then described in terms of a set of generalized coordinates $\mathbf{q}(t)$ which characterize the 3 translations and the 3 rotations of the frame $\mathfrak{R}_{e}$ attached to the end-effector with reference to the global frame $\mathfrak{R}_{G}$ (Fig. 3).

The DSMs of all the cables have to be assembled to form the oscillating model. In Section 2, the DSM of an inclined sagging cable $\mathbf{K}_{\text {dyn }}^{3 \mathrm{D}}$ (Eq. (19)) is expressed in the global cable frame $\mathfrak{R}_{i}$ relatively to the force-displacement response at the attachment point $A_{i}$. In order to assemble the DSMs of all the cables to formulate the overall system DSM, it is necessary to transform each DSM to the global frame $\mathfrak{R}_{G}$ : 


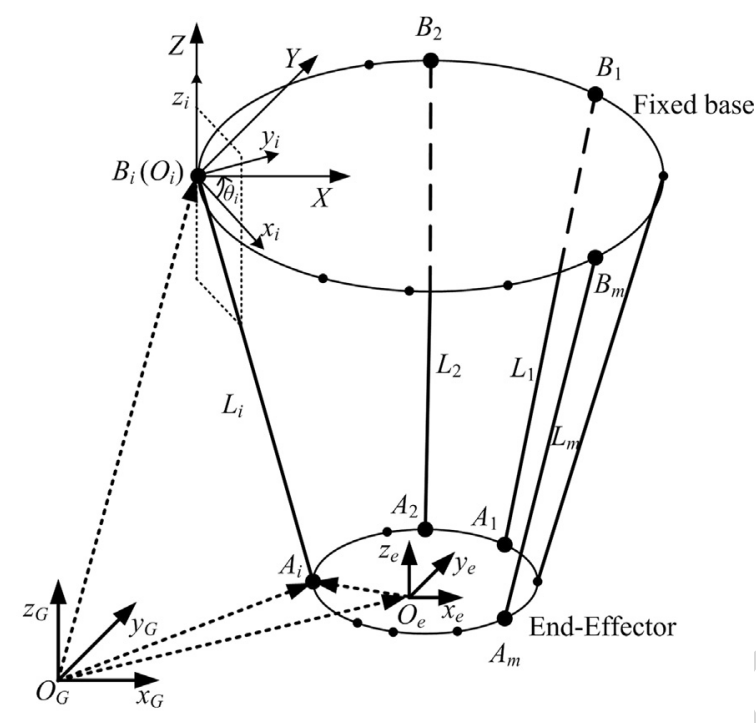

Global coordinate

Fig. 3. The schematic diagram of a suspended CDPR.

$$
{ }^{G} \mathbf{K}_{i}(\omega)={ }^{G} \mathbf{T}_{i}^{\mathrm{i}} \mathbf{K}_{\mathrm{dyn}}^{3 \mathrm{D} G} \mathbf{T}_{\mathrm{i}}^{-1},
$$

by using suitable $3 \times 3$ rotation matrices ${ }^{G} \mathbf{T}_{i}$. Then, the $6 \times 6$ DSM of the $\operatorname{CDPR}{ }^{G} \mathbf{K}_{E}(\omega)$ can be formulated by assembling the DSMs of the $m$ driving cables:

$$
\begin{aligned}
& { }^{G} \mathbf{K}_{E}(\omega)=\sum_{i=1}^{m} \mathbf{A}_{i}^{T G} \mathbf{K}_{i}(\omega) \mathbf{A}_{i}, \\
& \text { with: } \mathbf{A}_{i}=\left[\begin{array}{cccccc}
1 & 0 & 0 & 0 & z \overrightarrow{O_{e} A_{i}} & -y \overrightarrow{O_{e} A_{i}} \\
0 & 1 & 0 & -z \overrightarrow{O_{e} A_{i}} & 0 & x \overrightarrow{O_{e} A_{i}} \\
0 & 0 & 1 & y \overrightarrow{O_{e} A_{i}} & -x \overrightarrow{O_{e} A_{i}} & 0
\end{array}\right],
\end{aligned}
$$

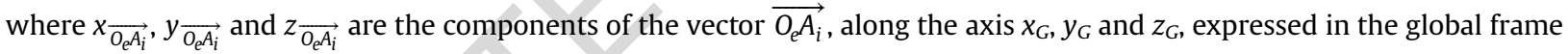
$\mathfrak{R}_{G}$. $O_{e}$ is the origin of the end-effector frame $\mathfrak{R}_{e}$ and $A_{i}$ is the point where the $i$ th cable is attached to the end-effector. In addition, the DSM of CDPRs can also be expressed in the end-effector frame $\mathfrak{R}_{e}$ through the coordinate transformation:

$$
{ }^{e} \mathbf{K}_{E}(\omega)=\left[\begin{array}{cc}
{ }^{e} \mathbf{T}_{G} & 0 \\
0 & { }^{e} \mathbf{T}_{G}
\end{array}\right] \cdot{ }^{G} \mathbf{K}_{E}(\omega) \cdot\left[\begin{array}{cc}
{ }^{e} \mathbf{T}_{G} & 0 \\
0 & { }^{e} \mathbf{T}_{G}
\end{array}\right]^{-1} .
$$

where ${ }^{e} \mathbf{T}_{G}$ is the rotation matrix that maps coordinates in the global frame to coordinates in the end-effector frame.

As explained in Section 2, the DSM of an inclined sagging cable is frequency-dependent because it considers the effect of cable mass on the cable dynamics. The DSM of a CDPR is also frequency-dependent, which means that each element of ${ }^{G} \mathbf{K}_{E}(\omega)$ depends on the frequency $\omega$ of the forcing function. This DSM will be used in the following CDPR dynamic model.

\subsection{Vibration model of the end-effector around a static equilibrium}

The oscillating model of the end-effector around a static equilibrium can be derived in terms of generalized coordinates by using the Lagrange's equations [49]:

$$
\frac{d}{d t}\left(\frac{\partial T}{\partial \dot{q}_{i}}\right)-\frac{\partial T}{\partial q_{i}}+\frac{\partial V}{\partial q_{i}}=f_{i}, i=1,2, \cdots 6
$$

where:

- $T$ and $V$ represent the kinetic and potential energies of the system, respectively;

- $q_{i}, i=1, \ldots, 6$, are the generalized coordinates corresponding to the 3 translational motions along axis- $x_{e}, y_{e}$ and $z_{e}$ and the 3 rotational motions around axis- $x_{e}, y_{e}$ and $z_{e}$. For example, in Section $4, q_{i}, i=1, \ldots, 3$ are the end-effector frame 
position coordinates $x, y, z$, and $q_{i}, i=4, \ldots, 6$ are the three parameters $\alpha, \beta, \gamma$ of the infinitesimal rotation vector defining the end-effector frame orientation in the global frame $\mathfrak{R}_{G}$.

- $\dot{q}_{i}$ is the time derivative of the generalized coordinate $q_{i}$;

- $f_{i}$ represents the non-conservative generalized force or moment applied to the end-effector.

According to the oscillating theory [50], the movements of the end-effector around its static equilibrium are assumed to be small, and the Coriolis acceleration can be neglected. Consequently, the oscillating model of the end-effector can be linearized by simplifying the kinetic energy $T$ of the system:

$$
T=\frac{1}{2} \dot{\mathbf{q}}^{T \mathbf{e}} \mathbf{M}_{\mathbf{E}} \dot{\mathbf{q}},
$$

where ${ }^{\mathbf{e}} \mathbf{M}_{\mathbf{E}}$ is the $6 \times 6$ generalized mass matrix of the end-effector expressed in frame $\mathfrak{R}_{e}$ (Eq. (26)):

$$
{ }^{\mathbf{e}} \mathbf{M}_{\mathbf{E}}=\left[\begin{array}{cc}
m_{E} \cdot \operatorname{diag}(1,1,1) & \mathbf{0} \\
\mathbf{0} & { }_{\mathbf{I}} \mathbf{I}_{\mathbf{E}}
\end{array}\right]
$$

$m_{E}$ is the mass of the end-effector and ${ }^{\mathbf{e}} \mathbf{I}_{\mathbf{E}}$ its $3 \times 3$ inertia matrix point $O_{e}$ and expressed in the frame $\mathfrak{R}_{e}$. $\mathbf{q}$ and $\dot{\mathbf{q}}$ are the column vectors of the generalized coordinates and their time derivatives: $\mathbf{q}=\left[q_{1} q_{2} q_{3} q_{4} q_{5} q_{6}\right]^{T}$ and $\dot{\mathbf{q}}=\left[\dot{q}_{1} \dot{q}_{2} \dot{q}_{3} \dot{q}_{4} \dot{q}_{5} \dot{q}_{6}\right]^{T}$.

The potential energy $V$ of the system is:

$$
V=\frac{1}{2} \mathbf{q}^{T^{e}} \mathbf{K}_{E} \mathbf{q} \text {. }
$$

According to Eq. (24), the dynamic equations of the end-effector of a CDPR around a static equilibrium can then be written in matrix form as:

$$
{ }^{\mathrm{e}} \mathbf{M}_{\mathbf{E}} \ddot{\mathbf{q}}(\mathbf{t})+{ }^{\mathrm{e}} \mathbf{K}_{\mathbf{E}}(\omega) \mathbf{q}(\mathbf{t})=\mathbf{f}(\mathbf{t}),
$$

where $\mathbf{f}(t)$ is a column vector of the non-conservative forces and moments: $\mathbf{f}(t)=\left[f_{1}(t) f_{2}(t) f_{3}(t) f_{4}(t) f_{5}(t) f_{6}(t)\right]^{T}$.

\subsection{Dynamic analysis of CDPRs}

When the effect of cable mass on the cable dynamics is neglected in the vibration analysis, the stiffness matrix ${ }^{{ }^{e}} \mathbf{K}_{\mathbf{E}}$ is constant and independent of the frequency $\omega$. According to the free vibration theory of multi-degree-of-freedom systems, the eigenfrequencies of the CDPR can then be calculated by transforming the system dynamic equations into its modal space, and then solving the classic eigenvalue and eigenvector problems [33].

In this paper, both the cable elasticity and the effect of cable mass on the cable dynamics are considered through the dynamic stiffness of CDPRs. Thereby, the internal degrees of freedom of the cables do not have to be considered. Each cable is reduced to an equivalent spring element whose nodal coordinates are the coordinates of the end-effector. The dynamic stiffness matrices of the cables become the basic matrices for assembling the global DSM of the CDPR. The order of the resulting eigenvalue equation of the system (28) is equal to the number of generalized coordinates of the CDPR and is frequency dependent because of ${ }^{e} \mathbf{K}_{\mathbf{E}}(\omega)$. Thus, the number of modes predicted by this model is not limited to the number of degrees of freedom of the CDPR. The classic modal analysis methods such as the modal superposition are no longer suitable because of the frequency dependence of the equation system. Hence, a suitable eigensolution procedure should be adopted to identify the eigenfrequencies.

In fact, one of the most important objective of modal analysis is to establish and verify, through vibration tests, an acceptable mathematical model that describes the dynamic behavior of the studied system. In this paper, modal superposition is not suitable because of the frequency dependence of ${ }^{{ }^{e}} \mathbf{K}_{\mathbf{E}}$ in Eq. (28). Hence, an original method to analyze the cable modes and their effect on the end-effector vibrations is presented. The FRFs of the CDPR to a harmonic excitation are used.

For each pose of the end-effector in the workspace, according to Eq. (28), the dynamic equations of a CDPR under a harmonic excitation can be written as:

$$
{ }^{\mathrm{e}} \mathbf{M}_{\mathbf{E}} \ddot{\mathbf{q}}(\mathbf{t})+{ }^{\mathrm{e}} \mathbf{K}_{\mathbf{E}}(\omega) \mathbf{q}(\mathbf{t})=\mathbf{f}(\mathbf{t}),
$$

where $\mathbf{f}(t)$ is a column vector of the harmonic excitation. This harmonic excitation can be expressed as:

$$
\mathbf{f}(t)=\overline{\mathbf{f}} e^{j \omega t},
$$

where $\overline{\mathbf{f}}$ is the column vector whose elements are complex numbers representing the amplitudes and the initial phases of the excitation.

Based on the assumption that the system can be considered time invariant during the experiments, if a harmonic excitation is inputed into the system at a given frequency, the system will respond at the same frequency with a certain magnitude and a certain phase angle relative to the input. The harmonic response of the end-effector $\mathbf{q}(t)$ can then be expressed as: 




Fig. 4. A schematic of the CDPR prototype CoGiRo [43].

$$
\mathbf{q}(t)=\overline{\mathbf{q}} e^{j \omega t} .
$$

Substituting Eqs. (30) and (31) into Eq. (29) yields:

$$
-{ }^{\mathbf{e}} \mathbf{M}_{\mathbf{E}} \omega^{2} \overline{\mathbf{q}} \mathbf{e}^{j \omega t}+{ }^{\mathbf{e}} \mathbf{K}_{\mathbf{E}}(\omega) \overline{\mathbf{q}} \mathbf{e}^{j \omega t}=\overline{\mathbf{f}} \mathbf{e}^{j \omega t} .
$$

The FRF matrix can then be expressed as:

$$
\mathbf{H}(\omega)=\frac{\mathbf{q}(t)}{\mathbf{f}(t)}=\frac{\overline{\mathbf{q}}}{\overline{\mathbf{f}}}=\frac{1}{-\omega^{2^{e}} \mathbf{M}_{E}+{ }^{e} \mathbf{K}_{E}(\omega)} .
$$

The FRF representation is the transfer function evaluated along the $j \omega$ frequency axis. In the above equations, the system responses $\mathbf{q}(t)$ are displacement responses and $\mathbf{H}(\omega)$ is also called the dynamic compliance. Generally, velocity responses and acceleration responses can also be used in the vibration analysis. The velocity responses $\mathbf{v}(t)$ and the acceleration responses a $(t)$ can be expressed as:

$$
\begin{aligned}
& \mathbf{v}(t)=\dot{\mathbf{q}}(t)=j \omega \overline{\mathbf{q}} e^{j \omega t}=\overline{\mathbf{v}} e^{j \omega t}, \\
& \mathbf{a}(t)=\ddot{\mathbf{q}}(t)=-\omega^{2} \overline{\mathbf{q}} e^{j \omega t}=\overline{\mathbf{a}} e^{j \omega t},
\end{aligned}
$$

where $\overline{\mathbf{v}}$ and $\overline{\mathbf{a}}$ are the column vectors whose elements are complex numbers representing the amplitudes and the initial phases of the velocity and acceleration responses such that: $\overline{\mathbf{v}}=j \omega \overline{\mathbf{q}}$ and $\overline{\mathbf{a}}=-\omega^{2} \overline{\mathbf{q}}$. According to Eq. (33), the FRFs of the end-effector under a harmonic excitation can be expressed as:

$$
\begin{aligned}
& \mathbf{H}_{V}(\omega)=\frac{\mathbf{v}(t)}{\mathbf{f}(t)}=\frac{\overline{\mathbf{v}}}{\overline{\mathbf{f}}}=\frac{j \omega}{-\omega^{2 \mathbf{e}} \mathbf{M}_{\mathbf{E}}+{ }^{\mathbf{e}} \mathbf{K}_{\mathbf{E}}(\omega)}, \\
& \mathbf{H}_{A}(\omega)=\frac{\mathbf{a}(t)}{\mathbf{f}(t)}=\frac{\overline{\mathbf{a}}}{\overline{\mathbf{f}}}=\frac{-\omega^{2}}{-\omega^{2} \mathbf{e} \mathbf{M}_{\mathbf{E}}+{ }^{\mathbf{e}} \mathbf{K}_{\mathbf{E}}(\omega)},
\end{aligned}
$$

where $\mathbf{H}_{V}(\omega)$ and $\mathbf{H}_{A}(\omega)$ are also called the mobility and the impedance, respectively. The dynamic information of a CDPR contained in these FRFs include the cable modes, the end-effector vibrations, and their coupling. In addition, the dynamic responses of the end-effector can be plotted as functions of the frequency $\omega$. From these plots, the resonances of the CDPR can be identified and the effect of cable dynamics on the CDPR vibrations can be analyzed. The latter issue will be further detailed in the following section.

\section{Experimental validations and discussion}

Dynamic experiments around two static equilibria and along a trajectory have been carried out on CoGiRo, a 6-DOF CDPR prototype suspended by 8 cables [43].

\subsection{Description of the CDPR prototype}

The schematic of CoGiRo is shown in Fig. 4. The configuration parameters are listed in Table 1 . This robot uses $\phi 4$ mm anti-rotation steel cables as driving cables. The Young's Modulus of the cable is $20 \mathrm{GPa}$ and the linear weight of the cable is 
Table 1

Configuration parameters of CoGiRo: coordinates of $B_{i}$ expressed in the global frame $\Re_{G}$, and coordinates of $A_{i}$ expressed in the end-effector frame $\mathfrak{R}_{e}$.

\begin{tabular}{|c|c|c|c|c|c|c|c|}
\hline$B_{i}$ & $x(\mathrm{~m})$ & $y(\mathrm{~m})$ & $z(\mathrm{~m})$ & $A_{i}$ & $x(\mathrm{~m})$ & $y(\mathrm{~m})$ & $z(\mathrm{~m})$ \\
\hline$B_{1}$ & -7.224 & -5.359 & 5.468 & $A_{1}$ & 0.500 & -0.507 & 0.555 \\
\hline$B_{2}$ & -7.435 & -5.058 & 5.477 & $A_{2}$ & -0.488 & 0.361 & 0.554 \\
\hline$B_{3}$ & -7.425 & 5.196 & 5.486 & $A_{3}$ & -0.500 & -0.260 & 0.555 \\
\hline$B_{4}$ & -7.210 & 5.497 & 5.495 & $A_{4}$ & 0.503 & 0.342 & 0.548 \\
\hline$B_{5}$ & 7.139 & 5.463 & 5.481 & $A_{5}$ & -0.500 & 0.507 & 0.555 \\
\hline$B_{6}$ & 7.440 & 5.158 & 5.494 & $A_{6}$ & 0.497 & -0.353 & 0.554 \\
\hline$B_{7}$ & 7.415 & -5.089 & 5.481 & $A_{7}$ & 0.499 & 0.260 & 0.549 \\
\hline$B_{8}$ & 7.113 & -5.388 & 5.492 & $A_{8}$ & -0.495 & -0.333 & 0.554 \\
\hline
\end{tabular}

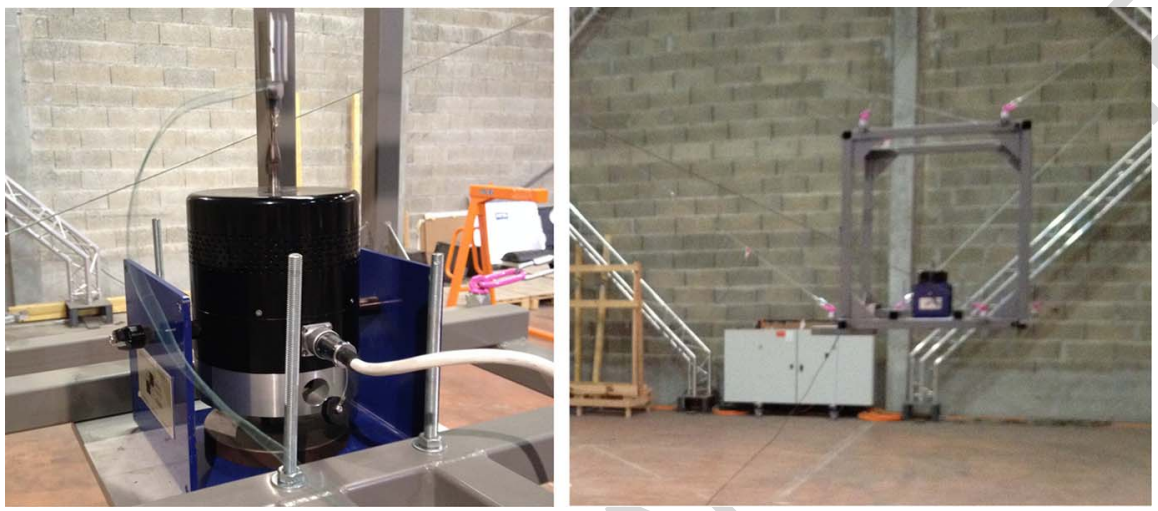

Fig. 5. Electro-dynamic shaker mounted on the end-effector of CoGiRo.

$0.067 \mathrm{~kg} / \mathrm{m}$. The Young's modulus has been identified using a material testing machine. All cables work within their linear elastic region. More details on this robot can be found in [43].

\subsection{Dynamic experiments around a static equilibrium}

The dynamic experiments aims at verifying the ability of the DSM method to find the eigen-properties of the CDPR prototype by:

- Identifying the eigenfrequencies of the driving cables and the resonances of the end-effector under harmonic excitation at certain poses in the workspace;

- Studying the interaction between local cable modes and global rigid-body oscillations of the end-effector.

\subsubsection{Experimental setup}

The experimental setup is shown in Fig. 5. An electro-dynamic shaker is mounted on the end-effector (PCB $\mathrm{K} 2100 \mathrm{E} 11 \pm 440 \mathrm{~N})$. A small mass block is fixed to the mobile stick of the shaker $(0.05 \mathrm{~kg})$. Thus, the shaker can deliver a harmonic vertical force to the end-effector. The force is proportional to the acceleration of the mass block, and can be measured by a force sensor placed between the mass block and the shaker mobile stick (PCB 208C05). A triaxial accelerometer fixed on the platform is used to obtain the responses of the end-effector along three mutually perpendicular directions (PCB 356A15). Moreover, one tri-axial accelerometer is fixed on each cable. A preliminary study allowed us to find where to fix the sensor to avoid particular wave nodes of transverse vibration which might have weakened the measured acceleration level. Each sensor has a mass of $0.0058 \mathrm{~kg}$. Compared to the weight of the cable taken as a whole (for example, for the 5 th cable, more than $0.4 \mathrm{~kg}$ at the center pose and more than $0.15 \mathrm{~kg}$ at the boundary pose, these center and boundary poses being defined below), it was assumed to be negligible. This assumption has not been verified further. The responses of the sensors are recorded by a National Instrument ${ }^{2}$ data acquisition system (NI - 9234).

During the experiments, the end-effector is firstly moved vertically from its home pose to a pose above the ground. The coordinates of the latter, measured by means of a laser tracker, are $x=0.012 \mathrm{~m}, y=0.0697 \mathrm{~m}, z=1.219 \mathrm{~m}$, and $\alpha, \beta, \gamma=0{ }^{\circ}$. This pose, referred to as the center pose, is used to check the validity of the model since it possesses a practically symmetrical arrangement of the cables. After all the cables and the end-effector are stabilized, a harmonic excitation is generated by the shaker. The frequency of the harmonic excitation is changed step by step. The step size is $0.05 \mathrm{~Hz}$. At each step, there is a

\footnotetext{
${ }^{2}$ http://www.ni.com/data-acquisition/.
} 


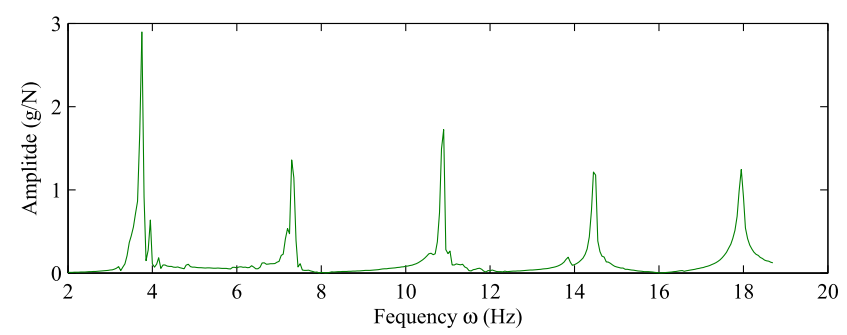

(a) FRF between the cable acceleration response and the excitation force

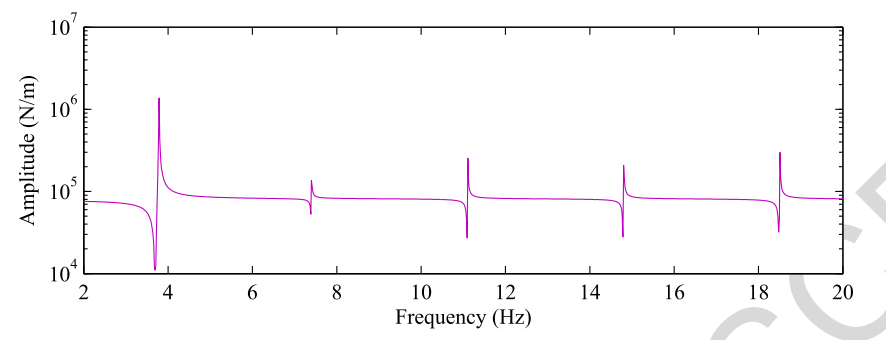

(b) Amplitude variation of the trace of the cable dynamic stiffness matrix obtained by the DSM method

Fig. 6. Experimental and simulation results of the vibration analysis of the 5th cable at the center pose $(x=0.012 \mathrm{~m} ; y=0.0697 \mathrm{~m} ; z=1.219 \mathrm{~m}$; $\left.\alpha, \beta, \gamma=0^{\circ}\right)$.

stabilization time of $8 \mathrm{~s}$, a measuring time of $8 \mathrm{~s}$, and a sample frequency of $1024 \mathrm{~Hz}$. The frequency range is chosen based on a sensitivity analysis of model output. Its range of interest is limited to $20 \mathrm{~Hz}$ in this work, even if the experimental tests were performed up to $30 \mathrm{~Hz}$. Indeed, it was found that the system is characterized by interactions between the cables modes and the rigid-body modes of the end-effector which are dominant in the low frequency range below $20 \mathrm{~Hz}$. As the electrodynamic shaker is not suitable for the frequency generation below $2 \mathrm{~Hz}$, the frequency range of interest is $2-20 \mathrm{~Hz}$.

The end-effector is then moved to another pose located near the workspace boundary $(x=4.012 \mathrm{~m} ; y=0.0697 \mathrm{~m}$; $z=1.219 \mathrm{~m} ; \alpha, \beta, \gamma=0^{\circ}$ ), and the previous procedure is repeated. This particular pose has been chosen for its asymmetric configuration in terms of cable lengths and force distribution in the cables.

\subsubsection{Experimental results and discussions: eigenfrequencies of the cables}

The cable dynamics are firstly analyzed to show the relevance of the dynamic stiffness matrix in reflecting the effects of cable modes on the dynamic behavior of the CDPR.

Fig. 6a presents the FRF plot of the 5th cable when the end-effector is at the center pose (Fig. 4). The 5th cable is chosen to illustrate the approach because it has the greater variation in length between the two studied poses. To assess the frequencies of the in-plane and out-of-plane cable modes, the FRF measurement shown in Fig. 6a refers to the norm of the acceleration vector and to the excitation force applied by the shaker mounted on the end-effector. The norm of the acceleration vector is effectively used to overcome the lack of precision in the alignment of the axes of the tri-axial accelerometer with those of the cable. The horizontal axis corresponds to the frequency range of $2-20 \mathrm{~Hz}$ while the vertical axis is the amplitude of the FRF. As the unit of acceleration used is $g$ and the unit of force is $N$, the unit of the amplitude is $g / N$. This FRF shows several sharp resonance peaks which correspond to the first eigenfrequencies of the cable.

The DSM method does not allow the computation of the FRF between the acceleration response of the cable at the measurement point and the force applied on the end-effector by the shaker. In fact, each cable is reduced to an equivalent spring element whose nodal coordinates are the coordinates of the end-effector. The internal degrees of freedom of the cables are thus not included in the model. A direct FRF-based model updating technique cannot thus be used to validate the DSM model. In order to demonstrate the procedure and to show the efficiency of the method to introduce the cable dynamics in the CDPR oscillating model, the amplitude of the trace of the cable DSM (Eq. (19)) is used for identifying the local cable modes in simulation, as shown in Fig. $6 \mathrm{~b}$. Note that the trace is invariant with respect to a change of basis and that the calculation of the cable tensions at the static equilibrium of the CDPR is done by a suitable force distribution algorithm [51]. As shown in Fig. 6b, several peaks appear. The corresponding changes in the cable stiffness are due to the cable modes and they illustrate that the DSM method reveals the effects of cable mass and elasticity on the dynamic behavior of the CDPR. Similar experiments and simulations were made at the boundary pose. The corresponding results are shown in Fig. 7.

As a result, the eigenfrequencies of the 5th cable can, on the one hand, be identified from the FRF measurements and, on the other hand, be predicted using the DSM model. The eigenfrequencies between 2 and $20 \mathrm{~Hz}$ are given in Table 2. According to this table, the eigenfrequencies of the cable modes identified by experimental analysis are close to those obtained by the DSM method for the two studied poses. The relative differences between simulation and experiment are all less than 


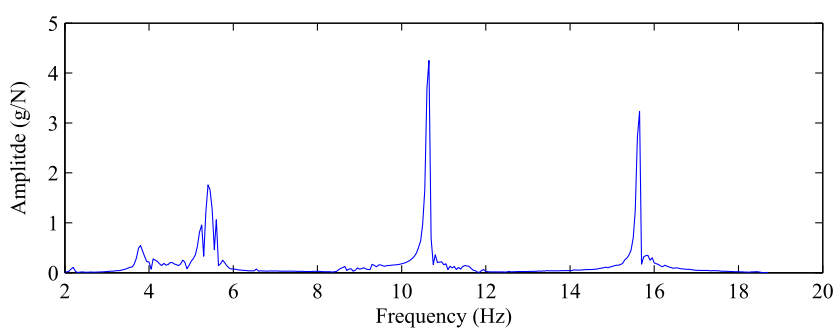

(a) FRF between the cable acceleration response and the excitation force



(b) Amplitude variation of the trace of the cable dynamic stiffness matrix obtained by the DSM method

Fig. 7. Experimental and simulation results of the vibration analysis of the 5th cable at the boundary pose $(x=4.012 \mathrm{~m} ; y=0.0697 \mathrm{~m} ; z=1.219 \mathrm{~m}$; $\left.\alpha, \beta, \gamma=0^{\circ}\right)$.

Table 2

Eigenfrequencies of the 5th cable (Fig. 4) between 2 and $20 \mathrm{~Hz}$ (identified from FRF measurements and computed using the DSM model).

\begin{tabular}{lll}
\hline$(\mathrm{Hz})$ & Center pose & Boundary pose \\
\hline$f_{1}$ meas. & 3.8 & 5.2 \\
$f_{1}$ calc. & 3.6 & 5.4 \\
$f_{2}$ meas. & 7.3 & 10.4 \\
$f_{2}$ calc. & 7.1 & 10.7 \\
$f_{3}$ meas. & 10.9 & 15.6 \\
$f_{3}$ calc. & 10.7 & 15.7 \\
$f_{4}$ meas. & 14.5 & - \\
$f_{4}$ calc. & 14.3 & - \\
$f_{5}$ meas. & 18.0 & - \\
$f_{5}$ calc. & 17.8 & - \\
\hline
\end{tabular}

$5 \%$. In the short-length configuration at the pose located near the workspace boundary, the increase of the cable tension strengthen the first natural modes and spread them over the frequency range. Only three resonances remain between 2 and $20 \mathrm{~Hz}$.

In Fig. 7a, it can be noted that the lowest frequency peak close to $4 \mathrm{~Hz}$ is not captured by the DSM prediction. Due to the experimental setup constraints, the dynamic cable behavior is not looked in isolation but only in a complete CDPR configuration. The presence of the peak at $4 \mathrm{~Hz}$ is due to the interaction between the first rigid-body mode of the end-effector and the cable oscillations, as described in more detail in the following section. In fact, there is a strong coupling of cable modes and end-effector resonances in low frequencies, where a lot of energy is transferred to the cables, due to the harmonic excitation used in the present experiments (16 s by frequency step), at the frequency of the first global end-effector rigid-body mode. When combined with the phase distributions of the involved modes, this results in a decrease of the first cable resonance.

Similar measurements were made for two other cables (cables 4 and 6, see Fig. 4). For the sake of brevity, since the observations were the same, only the results of cable 5 are reported here.

The results confirm that the DSM model can accurately predict the cable modes in the frequency range of interest.

\subsubsection{Experimental results and discussions on the dynamic behavior of the CDPR}

The experimental and simulation results of the vibration analysis of the end-effector at the center pose are shown in Fig. 8 .

Fig. 8a depicts the experimental FRF plots referring to the acceleration responses of the end-effector along the $x$-, $y$-, $z$ - 




(a) Measurements

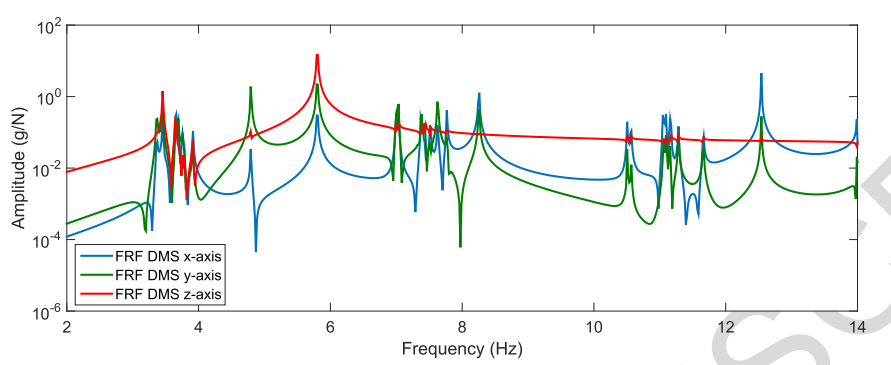

(b) DSM model

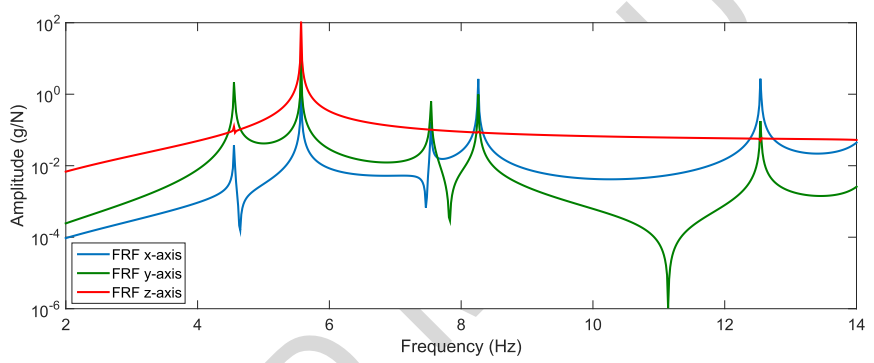

(c) Model with a constant static stiffness matrix

Fig. 8. Experimental and simulation results of the FRFs between the acceleration responses of the end-effector along the $x, y$, and $z$-axis and the excitation force along the $z$-axis at the center pose $\left(x=0.012 \mathrm{~m} ; y=0.0697 \mathrm{~m} ; z=1.219 \mathrm{~m} ; \alpha, \beta, \gamma=0^{\circ}\right)$.

axis and the excitation force along the $z$-axis. The corresponding FRFs computed from the DSM model by using Eq. (37) are given in Fig. 8b. As seen by comparing Fig. 8a and b, the DSM model considered in this paper does not provide a good fit for the FRF levels because the damping model used is too restrictive and does not depict the reality. To be practical, FRF-based model calibration requires an accurate identification of the damping mechanisms and of the spatial distribution of the damping over the system, in the presence of noisy data. Indeed, in this model, a constant viscous damping ratio of $1 \%$ is only used to describe the various damping mechanisms in steel wire cables such as hysteretic, viscous and viscoelastic which were recently identified [52]. According to a sensitivity analysis not presented here, a non-linear relationship between the cable axial damping and the frequency as well as some friction damping brought by the joints between the cables and the end-effector are also expected.

In the present study, it is not intended to carry out an in-depth investigation of the most appropriate damping model. The focus is placed on finding the correct number and values of the eigenfrequencies. It should be noted that estimation of modal damping ratios will have little effects in resonance identification. The damping related issue should be investigated in future works to better simulate the actual damping behavior.

From Fig. $8 \mathrm{~b}$, it is possible to identify in the studied frequency range numerous damped resonances of the end-effector suspended on the cable stiffness. The number of resonances is clearly not equal to the number of degrees of freedom of the system, i.e. to 6. The results obtained with the DSM model of the CDPR should be compared to the ones obtained with a model using a constant static stiffness matrix [33] (Fig. 8c). In the latter approach, the cable sag-induced stiffness and axial elasticity are considered but the effect of cable mass on the CDPR dynamics are neglected. This model predicts only the 6 rigid-body modes of the end-effector suspended on the static stiffness of the cables and omit the coupling between the dynamics of the platform and the cable resonances. A comparison of Fig. $8 \mathrm{~b}$ and c clearly shows that the dynamics of the cables changes the value of eigenfrequencies and add new resonances.

The complex interaction between local cable modes and global rigid-body oscillations of the end-effector can be analyzed by means of Fig. 9. This figure depicts the evolution of the 6 damped eigenvalues of the end-effector suspended on the 


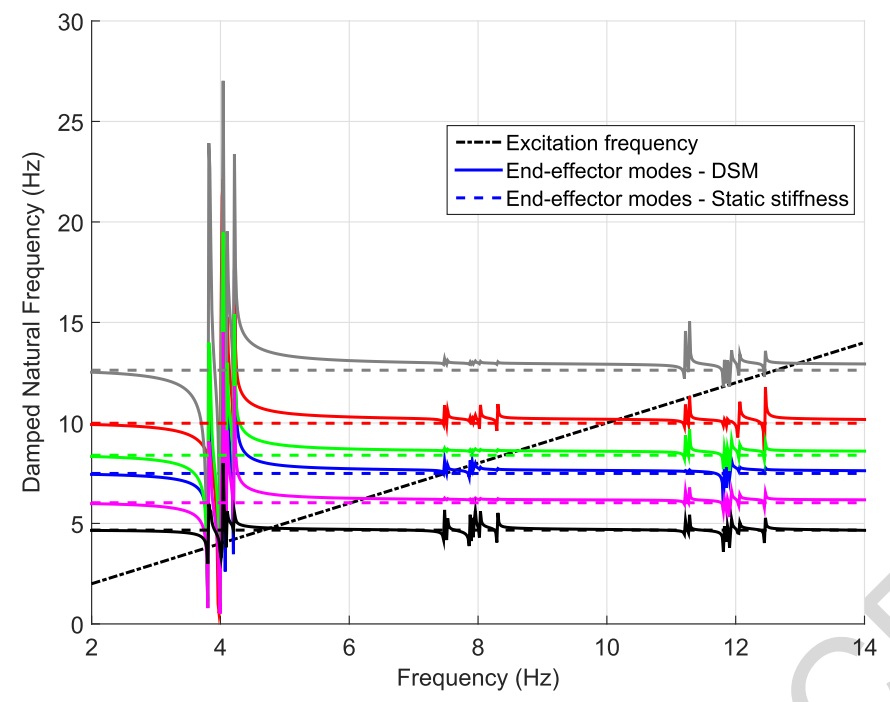

Fig. 9. Amplitude variations of the damped eigenfrequencies of the CDPR obtained by simulation at the center pose $(x=0.012 \mathrm{~m} ; y=0.0697 \mathrm{~m}$; $z=1.219 \mathrm{~m} ; \alpha, \beta, \gamma=0^{\circ}$ ).

cable stiffnesses over the frequency range $0-14 \mathrm{~Hz}$. The analytical model is developed in the frequency domain to represent the FRFs between any possible measurement locations and directions on the end-effector of the CDPR (Eq. (33)). The denominator of the FRF is the characteristic or frequency equation of the system, which depends on the frequency of the forcing function. The solution of this characteristic equation for a given frequency of the forcing function yields a set of 6 eigenvalues. The solid lines correspond to the damped eigenfrequencies computed at each frequency of the forcing function with the proposed model using the DSM. The eigenvalues are calculated and plotted with a frequency step of $0.05 \mathrm{~Hz}$. Comparing the solid lines with the dotted lines which represent the damped eigenfrequencies obtained with a model synthesized with a constant static stiffness matrix [33], it is apparent that the stiffness variations in the region of the cable modes modify the eigenvalues of the overall robot.

Cable dynamics affect the CDPR response when a periodic excitation source is directly or indirectly applied to the driving cables. The first bisector in Fig. 9 represents the frequency $\omega$ of the forcing function. Each intersection between the frequency curves (end-effector modes) and the first bisector (excitation frequency) should result in a resonance on the CDPR responses, depending upon the relative direction of the eigenvector and of the excitation force. Comparing Fig. 8a with Fig. 9, we can find out that the first strong resonance just below $4 \mathrm{~Hz}$ for each response direction of the FRFs is due to the first mode of the 8 driving cables. The changes in the cable stiffnesses lower locally the eigenvalues of the robot. Since the pose of the end-effector is at the center of the workspace, all the cables have similar lengths and tensions and, consequently, have similar first eigenfrequencies. The second cable modes (symmetric in-plane mode) and the third cable modes (antisymmetric in-plane mode) induce the numerous but relatively damped resonances around $8 \mathrm{~Hz}$ and $12 \mathrm{~Hz}$, respectively.

The two resonances of the end-effector response in the $y$-axis at 4.7 and $6 \mathrm{~Hz}$ are due to the two first rigid-body modes of the end-effector suspended on the stiffness of the cables (Fig. 8a). The associated eigenvectors of these two modes are close to the $y$-axis of the global frame. Neglecting the effect of the cable dynamics in the CDPR vibration analysis [33], these resonances due to the two first rigid-body modes of the end-effector are predicted at frequencies just below (Fig. 8c). It should be noted that the DSM model damps the cables modes considerably more than the rigid-body modes of the endeffector (Fig. 8b and c). The model should thus account separately for different transverse and axial damping parameters to better simulate the true damping behavior of steel wire cables.

It is shown that the method presented in this paper predicts more accurately all the eigensolutions of the measured FRF data. Experimentally, the eigenfrequencies are globally a little bit lower (about $0.5 \mathrm{~Hz}$ ). It should be due to the modeling and experimental errors. In the experimental setup, metal rings are used as joints to connect the cables to the end-effector. However, it is assumed in the simulation that the cable end is directly fixed to the end-effector. Another reason could be the inertia parameters of the end-effector used in the simulations. These parameters have been obtained by CAD model without considering the welds.

Similar experiments have been made at the boundary pose ( $\left.x=4.012 \mathrm{~m} ; y=0.0697 \mathrm{~m} ; z=1.219 \mathrm{~m} ; \alpha, \beta, \gamma=0^{\circ}\right)$, and the same conclusions were drawn. For the sake of brevity, the corresponding experimental results are not presented here.

\subsection{Trajectory experiments}

This section aims at studying the dynamic behavior of the CDPR prototype along a trajectory of its end-effector. Since the DSM model introduced in this paper does not allow the computation of the time-domain response, the analysis of 

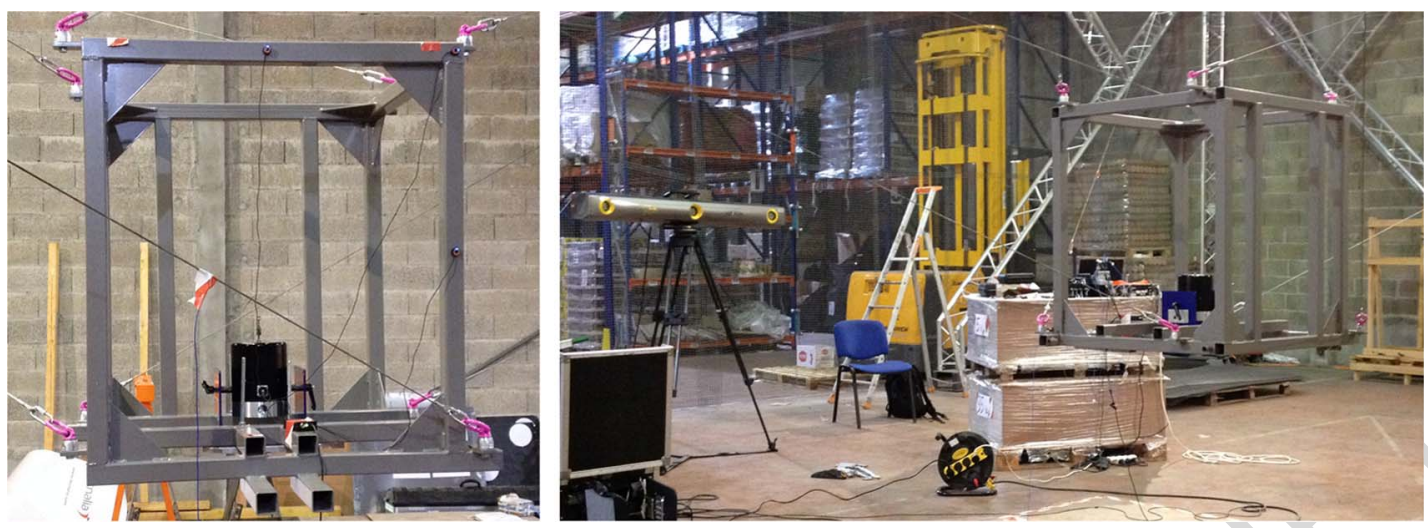

Fig. 10. Experimental setup used for the trajectory experiments.



(a) Pose error of the end-effector along the $y$-axis measured in the $x O y$ plane

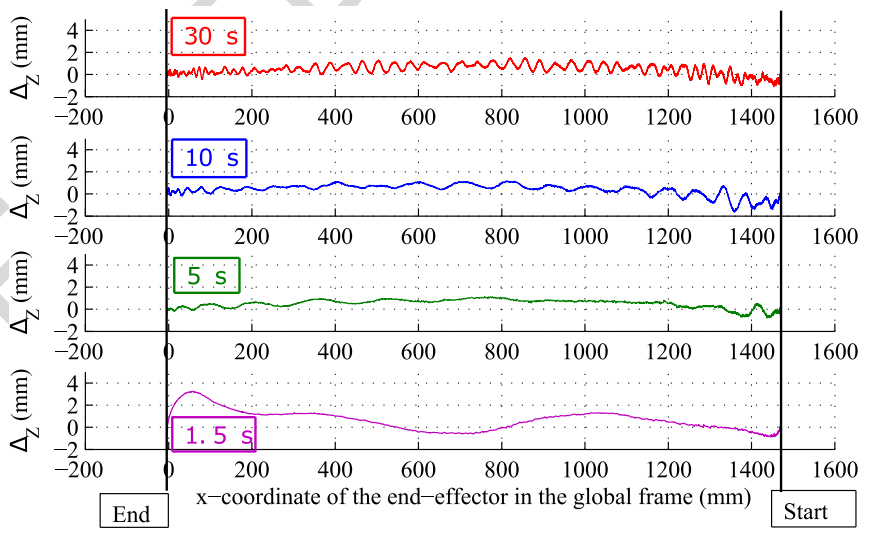

(b) Pose error of the end-effector along the $z$-axis measured in the $x O z$ plane

Fig. 11. Pose error of the end-effector during the trajectory from $x=1 \mathrm{~m} ; y=-2 \mathrm{~m} ; z=1.3 \mathrm{~m} ; \alpha, \beta, \gamma=0^{\circ}$ to $x=-0.5 \mathrm{~m} ; y=-0.7 \mathrm{~m} ; z=1.05 \mathrm{~m}$; $\alpha, \beta, \gamma=0^{\circ}$ with different trajectory durations.

experimental CDPR trajectories is done in the frequency domain focusing on the coupling between the cable dynamics and the end-effector vibrations.

The experimental procedure is described below.

1) As shown in Fig. 10, a photogrammetric Nikon Metrology K600-10 system ${ }^{3}$ based on three CCD linear cameras and infrared light active LEDs is used. Three LEDs are attached to the end-effector and their positions are measured simultaneously 


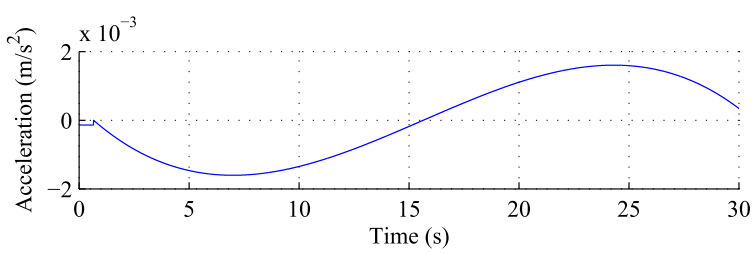

(a) Acceleration of the end-effector along the $z$-axis during the trajectory of 30 seconds (data given by the controller )

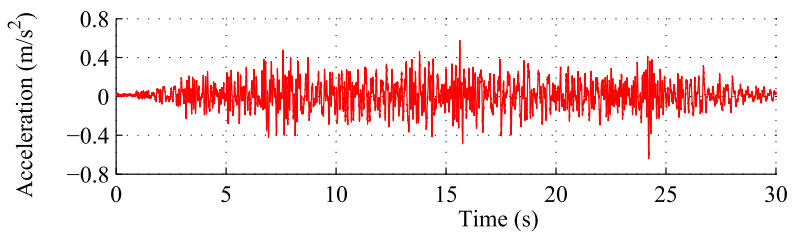

(b) Acceleration of the end-effector along the $z$-axis during the trajectory of 30 seconds (data measured by the accelerometer). These data do not contain the continuous component of the acceleration



(c) Time-Frequency response of the end-effector along the $z$-axis during the trajectory of 30 seconds

Fig. 12. Experimental results of the vibration analysis of the end-effector along the $z$-axis during the trajectory of $30 \mathrm{~s}$.

by the camera, thereby allowing the simultaneous tracking of multiple points and the measurement of both the position and the orientation of the end-effector. The system has a maximum position measuring accuracy of $37 \mu \mathrm{m}$ for a single point. An accelerometer is fixed along the cable, and a triaxial accelerometer is fixed to one edge of the end-effector.

2) The controller is asked to move the end-effector along a straight line trajectory from $x=1 \mathrm{~m}, y=-2 \mathrm{~m}, z=1.3 \mathrm{~m}$, $\alpha, \beta, \gamma=0^{\circ}$ to $x=-0.5 \mathrm{~m}, y=-0.7 \mathrm{~m}, z=1.05 \mathrm{~m}, \alpha, \beta, \gamma=0^{\circ}$. The time of this trajectory is set to be $30 \mathrm{~s}$ in the controller. Along the trajectory, the pose of the end-effector is measured by the Nikon system, and the measurements of the accelerometers is recorded by the National Instrument data acquisition system.

3) Once the motion is achieved, the controller is asked to move the end-effector back to the starting point of the trajectory. The same trajectory is then followed again with three other durations: 10, 5, and $1.5 \mathrm{~s}$.

Firstly, the pose error of the end-effector during the trajectory is studied. The trajectory in the controller is an ideal straight line. However, due to the vibrations of the end-effector, the real trajectory in the experiment is not a straight line. The pose error of the end-effector is defined as the difference between the desired end-effector pose and the end-effector pose measured by the Nikon system. Fig. 11 shows the pose error of the end-effector along the trajectory for the four different durations. Fig. 11a shows the pose error along the $y$-axis, and Fig. 11a shows the pose error along the $z$-axis. From Fig. 11, obvious variations of the pose error can be found. These variations are due to the vibrations of the end-effector. It is also found that the faster the end-effector moves, the larger the variation becomes. This means that the velocity and the acceleration of the end-effector can affect the amplitude of its vibrations.

\footnotetext{
${ }^{3}$ http://www.nikonmetrology.com/optical_cmm/.
} 
Moreover, time-frequency analysis of the acceleration measurements are made to study the frequency content of the vibrations of the end-effector during the trajectory. Fig. 12 presents the experimental results of the vibration analysis of the end-effector along the $z$-axis during the trajectory. The acceleration of the end-effector is shown in Fig. 12b, and the timefrequency response of the end-effector is shown in Fig. 12c. Firstly, in Fig. 12, the peaks of the vibration amplitude are seen to appear at $7 \mathrm{~s}, 15 \mathrm{~s}, 24 \mathrm{~s}$ during the trajectory. These peaks correspond to the extreme points and the inflection point of the acceleration curve. Secondly, three groups of frequencies can be identified from Fig. 12c: One group around $4 \mathrm{~Hz}$, a second group around $8 \mathrm{~Hz}$, and a third group around $12 \mathrm{~Hz}$. Referring to Fig. 9 in Section 4.2.1, these frequencies correspond to the end-effector eigenfrequencies affected by the cable modes.

\section{Conclusions and perspectives}

With the intention to evaluate the effect of the cable natural modes on CDPR vibrations, this paper focused on a dynamic stiffness analysis where the cable vibrations, the end-effector vibrations, and their coupling are taken into account. To this end, the calculations of FRFs were detailed. The changes in stiffness in the region of the cable eigenfrequencies affect the eigenfrequencies of the overall robot. This results in numerous system resonances which are relevant in cases such as CDPRs for wind tunnels and outdoor CDPR applications, where a periodic excitation source may be directly or indirectly applied. Moreover, the proposed vibration analysis methodology was illustrated by means of experiments performed on a large 6-DOF 8-cable CDPR prototype called CoGiRo. Experiments around two static equilibria and along a trajectory were reported. These experiments validate the vibration analysis methodology introduced in this paper, and allowed an analysis of the CoGiRo CDPR vibrations. The latter analysis revealed a strong coupling between cable and end-effector vibrations when a periodic external excitation is applied. Further experiments and analysis are however required in order to further investigate the damping mechanisms.

Based on the results of this paper, methods to reduce vibrations can be further developed, notably in order to significantly improve end-effector path following accuracy. For example, active vibration canceling or input shaping methods can be used to suppress or, at least, attenuate vibrations. Using the proposed system modeling and analysis method, any vibration mode may be eliminated by a suitable open-loop or closed-loop control strategy since it can be estimated with good accuracy. By extending attention to developments in cable-supported bridge dynamic analysis [53,54], further investigations should be dedicated to the potential impact of frequency veering and modal hybridization phenomena on the spectral properties of CDPRs.

\section{Acknowledgements}

We would like to thank Tecnalia for having allowed us to perform experiments on the CoGiRo CDPR prototype.

\section{References}

[1] J.-P. Merlet, Parallel Robots, Springer Science \& Business Media, vol. 128, 2006.

[2] D. Stewart, A platform with six degrees of freedom, Proc. Inst. Mech. Eng., 180 (1), 1965, pp. 371-386.

[3] R. Clavel, Conception d'un robot parallèle rapide à 4 degrés de liberté, Thèse de doctorat, École Polytechnique Fédérale de Lausanne, 1991.

[4] B. Zi, B. Duan, J. Du, H. Bao, Dynamic modeling and active control of a cable-suspended parallel robot, Mechatronics 18 (1) (2008) 1-12.

[5] P. Bosscher, R.L. Williams, M. Tummino, A concept for rapidly-deployable cable robot search and rescue systems, in: Proceedings of the ASME International Design Engineering Technical Conferences and Computers and Information in Engineering Conference, American Society of Mechanical Engineers, 2005, pp. 589-598.

[6] J.-P. Merlet, Marionet, a family of modular wire-driven parallel robots, in: Advances in Robot Kinematics: Motion in Man and Machine, Springer, 2010, pp. 53-61.

[7] S. Tadokoro, R. Verhoeven, M. Hiller, T. Takamori, A portable parallel manipulator for search and rescue at large-scale urban earthquakes and an identification algorithm for the installation in unstructured environments, in: Proceedings of the IEEE/RSJ International Conference on Intelligent Robots and Systems, IROS'99, vol. 2, 1999, pp. 1222-1227.

[8] Y. Mao, S.K. Agrawal, Design of a cable-driven arm exoskeleton (CAREX) for neural rehabilitation, IEEE Trans. Robot. 28 (4) (2012) $922-931$.

[9] G. Rosati, P. Gallina, S. Masiero, A. Rossi, Design of a new 5 DOF wire-based robot for rehabilitation, in: Proceedings of the 9th IEEE International Conference on Rehabilitation Robotics, ICORR, 2005, pp. 430-433.

[10] P. Gallina, G. Rosati, Manipulability of a planar wire driven haptic device, Mech. Mach. Theory 37 (2) (2002) 215-228.

[11] D. Surdilovic, J. GMP, R. Bernhardt, STRING-MAN: Wire-robot technology for safe, flexible and human-friendly gait rehabilitation, in: Proceedings of the 10th IEEE International Conference on Rehabilitation Robotics, ICORR, 2007, pp. 446-453.

[12] C. Gosselin, Stiffness mapping for parallel manipulators, IEEE Trans. Robot. Autom. 6 (3) (1990) 377-382.

[13] S. Kawamura, W. Choe, S. Tanaka, S.R. Pandian, Development of an ultrahigh speed robot falcon using wire drive system, in: Proceedings of the IEEE International Conference on Robotics and Automation, vol. 11995, pp. 215-220.

[14] S. Algermissen, R. Keimer, M. Rose, E. Breitbach, H.P. Monner, Applied robust control for vibration suppression in parallel robots, in: Proceedings of the 22nd International Symposium on Automation and Robotics in Construction (ISARC), Ferrara, Italy, 2005.

[15] R. Gexue, L. Qiuhai, H. Ning, N. Rendong, P. Bo, On vibration control with stewart parallel mechanism, Mechatronics 14 (1) (2004) 1-13.

[16] B. Kang, J.K. Mills, Vibration control of a planar parallel manipulator using piezoelectric actuators, J. Intell. Robot. Syst. 42 (1) (2005) 51-70.

[17] K. Kozak, I. Ebert-Uphoff, W. Singhose, Locally linearized dynamic analysis of parallel manipulators and application of input shaping to reduce vibrations, J. Mech. Des. 126 (1) (2004) 156-168.

[18] S. Mitsuta, E. Okawa, K. Seto, H. Ito, Active vibration control of structures arranged in parallel, JSME Int. J. Ser. C Dyn. Control Robot. Des. Manuf. 37 (3) 
(1994) 436-443.

[19] G. Piras, W. Cleghorn, J. Mills, Dynamic finite-element analysis of a planar high-speed, high-precision parallel manipulator with flexible links, Mech. Mach. Theory 40 (7) (2005) 849-862.

[20] M. Rognant, E. Courteille, P. Maurine, A systematic procedure for the elastodynamic modeling and identification of robot manipulators, IEEE Trans. Robot. 26 (6) (2010) 1085-1093.

[21] Y. Yun, Y. Li, Design and analysis of a novel 6-dof redundant actuated parallel robot with compliant hinges for high precision positioning, Nonlinear Dyn. 61 (4) (2010) 829-845.

[22] X. Zhang, J.K. Mills, W.L. Cleghorn, Vibration control of elastodynamic response of a 3-PRR flexible parallel manipulator using pzt transducers, Robotica $26(05)(2008) 655-665$.

[23] X. Diao, O. Ma, Vibration analysis of cable-driven parallel manipulators, Multibody Syst. Dyn. 21 (4) (2009) $347-360$.

[24] J. Du, H. Bao, C. Cui, D. Yang, Dynamic analysis of cable-driven parallel manipulators with time-varying cable lengths, Finite Elem. Anal. Des. 48 (1) (2012) 1392-1399.

[25] J. Du, W. Ding, H. Bao, Cable vibration analysis for large workspace cable-driven parallel manipulators, in: Cable-Driven Parallel Robots, Springer, 2013, pp. 437-449.

[26] O. Ma, X. Diao, Dynamics analysis of a cable-driven parallel manipulator for hardware-in-the-loop dynamic simulation, in: Proceedings of the IEEE/ ASME International Conference on Advanced Intelligent Mechatronics, 2005, pp. 837-842.

[27] A.F. Tang, Y. Li, L.F. Kong, X.J. Cheng, Vibration analysis of tendon-based parallel robot for processing, Adv. Mater. Res. 655 (2013) $1086-1091$.

[28] X. Weber, L. Cuvillon, J. Gangloff, Active vibration canceling of a cable-driven parallel robot using reaction wheels, in: Proceedings of the IEEE/RSJ International Conference on Intelligent Robots and Systems (IROS 2014), 2014, pp. 1724-1729.

[29] H. Yuan, E. Courteille, D. Deblaise, Static and dynamic stiffness analyses of cable-driven parallel robots with non-negligible cable mass and elasticity, Mech. Mach. Theory 85 (2015) 64-81.

[30] J. Du, S.K. Agrawal, Dynamic modeling of cable-driven parallel manipulators with distributed mass flexible cables, J. Vib. Acoust. 137 (2) (2015) 021020.

[31] S. Kawamura, H. Kino, C. Won, High-speed manipulation by using parallel wire-driven robots, Robotica 18 (01) (2000) $13-21$.

[32] T. Bruckmann, C. Sturm, W. Lalo, Wire robot suspension systems for wind tunnels, Wind Tunn. Exp. Fluid Dyn. Res. (2010) 29-50.

[33] K. Kozak, Q. Zhou, J. Wang, Static analysis of cable-driven manipulators with non-negligible cable mass, IEEE Trans. Robot. 22 (3) (2006) 425-433.

[34] E. Ottaviano, V. Gattulli, F. Potenza, Elasto-static model for point mass sagged cable-suspended robots, Adv. Robot Kinemat. (2016) 1-8.

[35] D.Q. Nguyen, M. Gouttefarde, O. Company, F. Pierrot, On the simplifications of cable model in static analysis of large dimension cable-driven parallel robots, in: Proceedings of the IEEE/RSJ International Conference on Intelligent Robots and Systems (IROS), 2013, pp. 928-934.

[36] J.-P. Merlet, On the inverse kinematics of cable-driven parallel robots with up to 6 sagging cables, in: Proceedings of the IEEE/RSJ International Conference Intelligent Robots and Systems (IROS), Hamburg, Germany, 2015, pp. 4356-4361.

[37] M. Nahon, Dynamics and control of a novel radio telescope antenna, in: Proceedings of the Modeling and Simulation Technologies Conference and Exhibit, American Institute of Aeronautics and Astronautics, 1999.

[38] Y. Ni, J. Ko, G. Zheng, Dynamic analysis of large-diameter sagged cables taking into account flexural rigidity, J. Sound Vib. 257 (2) (2002) 301-319.

[39] U. Starossek, Dynamic stiffness matrix of sagging cable, J. Eng. Mech. 117 (12) (1991) 2815-2828.

[40] J. Banerjee, Dynamic stiffness formulation for structural elements: a general approach, Comput. Struct. 63 (1) (1997) $101-103$.

[41] A. Leung, Dynamic stiffness method for exponentially varying harmonic excitation of continuous systems, J. Sound Vib. 98 (3) (1985) 337-347.

[42] T. Richards, Y. Leung, An accurate method in structural vibration analysis, J. Sound Vib. 55 (3) (1977) $363-376$.

[43] M. Gouttefarde, J.-F. Collard, N. Riehl, C. Baradat, Geometry selection of a redundantly actuated cable-suspended parallel robot, IEEE Trans. Robot. 31 (2) (2015) 501-510.

[44] A. Ansell, The dynamic element method for analysis of frame and cable type structures, Eng. Struct. 27 (13) (2005) $1906-1915$.

[45] J. Kim, S.P. Chang, Dynamic stiffness matrix of an inclined cable, Eng. Struct. 23 (12) (2001) 1614-1621.

[46] R.J. Allemang, Experimental Modal Analysis for Vibrating Structures.

[47] U. Starossek, Boundary Induced Vibration and Dynamic Stiffness of a Sagging Cable, ISD Universität Stuttgart, 1991

[48] H.M. Irvine, Free vibrations of inclined cables, J. Struct. Div. 104 (2) (1978) 343-347.

[49] S.S. Rao, F.F. Yap, Mechanical Vibrations, Addison-Wesley, New York, 1995.

[50] D. Bellet, Cours de Mécanique Générale, Cepadues-editions, 1988.

[51] H. Yuan, E. Courteille, D. Deblaise, Force distribution with pose-dependent force boundaries for redundantly actuated cable-driven parallel robots, J. Mech. Robot. 8 (4) (2016) 041004.

[52] M. Miyasaka, M. Haghighipanah, Y. Li, B. Hannaford, Hysteresis model of longitudinally loaded cable for cable driven robots and identification of the parameters, in: Proceedings of the IEEE International Conference on Robotics and Automation (ICRA), 2016, pp. 4051-4057.

[53] V. Gattulli, M. Lepidi, Localization and veering in the dynamics of cable-stayed bridges, Comput. Struct. 85 (21) (2007) 1661-1678.

[54] M. Lepidi, V. Gattulli, Non-linear interactions in the flexible multi-body dynamics of cable-supported bridge cross-sections, Int. J. Non-Linear Mech. 80 (2016) 14-28. 